\title{
Benign Prostatic Hyperplasia (BPH) Treatment with Physiotherapeutic Device/Thermobalancing Therapy causes New Challenges in Watchful Waiting
}

\author{
Ivan Gerasimovich Aghajanyan ${ }^{1}$ and Simon Allen ${ }^{2 *}$ \\ ${ }^{1}$ Head of the Department of Urology at the Yerevan State Medical University, Republic of Armenia 9 Ezras Hasratyan St, Yerevan, 0052, Armenia, UK \\ ${ }^{2}$ Director of Fine Treatment, 29 Rewley Road, Oxford, OX1 2RA, United Kingdom
}

Received: 12 February, 2016; Accepted: 16 February, 2016; Published: 17 February, 2016

*Corresponding author: Allen Simon, MD-PhD, Director of Fine Treatment, 29 Rewley Road, Oxford, OX1 2RA, United Kingdom; Tel: +447958878300; Fax: +441865728255; E-mail: info@finetreatment.com

Keywords: BPH treatment; Prostate enlargement; Thermobalancing therapy; Prostate natural treatment; Lower urinary tract symptoms; Watchful waiting; LUTS; Enlarged prostate; Clinical trial.

Lower urinary tract symptoms/ benign prostatic hyperplasia - LUTS/ BPH occurs in almost all men as they age.The papers show that LUTS are common in the UK, affecting $\approx 3 \%$ of men aged 45 49 years, rising to $>30 \%$ in men aged $\geq 85$ years. European and USA studies have reported the major impact of LUTS on quality of life of the patient and their partner [1]. As this aging condition is public, LUTS/ BPH prevention or impeding of its progression has priority for researches. Epidemiological data show a favorable relationship between healthy eating habits and regular physical activity level and a lower risk for LUTS or progression of LUTS [2]. But nor healthy food neither exercises ever have protected men from the development of BPH.

There were investigations that prostatic inflammation represents an important factor in influencing prostatic growth and progression of symptoms [3]. The reports presented at a satellite symposium entitled, "Inflammation and Prostatic Diseases: From Bench to Bedside," held during the 2015 annual meeting of the European Association of Urology in Madrid, Spain were discussed the link between inflammation and BPH. Prostatic inflammation has been shown to increase by threefold the risk for acute urinary retention, an end point in the natural progression of BPH. Inflammation has been proposed as the common thread between the metabolic syndrome and $\mathrm{BPH} /$ LUTS, which frequently co-exist, and offers new therapeutic targets for medical treatment [4].

Dr. Allen's research on the Origin of Diseases has helped him to identify the causal root of prostate enlargement, namely capillary expansion. This conclusion is based on 2 functional physiological properties of capillaries which are activated by an irritating factor, i.e., a trigger; indeed this peculiarity of capillaries explains the cause of chronic internal diseases. Constriction of capillaries in response to an irritating trigger develops local micro-hypothermia. It is this focus of hypothermia, which in turn becomes a constant irritant maintaining illness, i.e., making a disease chronic. In response to irritation, i.e., a trigger-initiator (cold/ infection/ inflammation) and later focus of hypothermia, and in order to eliminate them, the blood flow increases through the spontaneous expansion of the capillary net locally. The formation of new capillaries is essentially the growth of the excess tissue [5].

Thermobalancing therapy is the only external non-invasive treatment that targets pathological nidus continuously for a prolonged period of time, i.e., for days, months or even years, maintaining the accumulated temperature. We believe that the use of therapeutic device by keeping the temperature in the projection of the prostate gland acts on micro-focus of hypothermia and ischemia in it, removing the vicious cycle of spontaneous growth of capillaries in response to a trigger 'microhypothermia', thereby relieving the pressure in prostate and easing the BPH symptoms [6].

A 2-yr clinical trial involving 124 men with $\mathrm{BPH}$, to whom the natural therapeutic device, termed Dr. Allen's Device, was administered as a mono-therapy and has confirmed the effectiveness and safety of Thermobalancing therapy. After a 6-month treatment with this device, the patients reported a significant improvement to the disturbing LUTS; the prostate volume reduced and the maximum urinary flow rate increased. At the same time the clinical parameters in the control group, in which men didn't receive any treatment, i.e., were in watchful waiting, worsened [7].

Watchful waiting is a well-known approach in the management of benign prostatic hyperplasia. However, other studies and randomized placebo-controlled trials have confirmed the progressive nature of $\mathrm{BPH}$, with symptom progression being the most common manifestation. Variables such as baseline 
prostate-specific antigen, prostate volume, symptom score and bother score, decreased flow rate, and increased postvoid residual have all been linked to progression to acute urinary retention and need for surgery [8].

So, what should be done? The recent study on a treatment satisfaction measure for LUTS/ BPH suddenly explored no statistically significant differences in treatment satisfaction with tamsulosin vs placebo [9]. And this BPH medication was used for decades. A substantial body of evidence exists which points to serious and potentially ill-health effects of $5 \alpha$-RIs' therapy, namely finasteride or dutasteride. These include loss or reduced libido, erectile dysfunction, orgasmic and ejaculatory dysfunction, development of high-grade PCa tumors, potential negative cardiovascular events, and depression [10].

Prostate surgical procedures are unsafe [11]. That is why EAU Guidelines on the Treatment and Follow-up of Non-neurogenic Male Lower Urinary Tract Symptoms Including Benign Prostatic Obstruction suggests: Prostate surgery is indicated in men with absolute indications or drug treatment-resistant LUTS due to benign prostatic obstruction [12].

\section{Conclusion}

The outcomes of our clinical trial suggest that thermobalancing therapy may play key role in the activation of 'watchful waiting' for LUTS/ BPH treatment, as it effective and cannot harm. Intervention with anti-inflammatory drugs for men in starting point of prostate enlargement must be considered carefully as theses medications have serious side effects. It may be an idea to study deeper the use of natural products that have anti-inflammatory properties, such as cranberry, Saw Palmetto, Siberian Ginseng and other, together with physiotherapeutic device in men with LUTS/ BPH.

\section{Competing Interests}

The authors declare that they have no competing interests.

\section{References}

1. Speakman M, Kirby R, Doyle S, Loannou C. Burden of male lower urinary tract symptoms (LUTS) suggestive of benign prostatic hyperplasia (BPH) - focus on the UK. BJU Int. 2015;115(4):508-519. doi: $10.1111 /$ bju. 12745 .

2. Lin PH, Freedland SJ. Lifestyle and LUTS: what is the correlation in men? Curr Opin Urol. 2015;25(1):1-5. doi: 10.1097/ MOU.0000000000000121.

3. Chughtai B, Lee R, Alexis Te, Steven Kaplan. Role of Inflammation in Benign Prostatic Hyperplasia. Rev Urol. 2011;13(3):147-150.

4. Nickel JC. Role of Prostatic Inflammation in the Clinical Presentation of Benign Prostatic Hyperplasia. European Urology Supplements. 2015;14(9):e1459-e1463.

5. Allen S, Adjani A. Therapeutic Device and Method, US 20110152986 A1. 2009; http://www.google.com/patents/US20110152986.

6. Aghajanyan IG Allen S. Positive Response to Thermobalancing Therapy Enabled by Therapeutic Device in Men with Non-Malignant Prostate Diseases: BPH and Chronic Prostatitis. Diseases. 2016;4:18.

7. Allen S, Aghajanyan IG. Benign Prostatic Hyperplasia Treatment with New Physiotherapeutic Device. Urol J. 2015;12(5):2371-2376.

8. Wiygul J, Babayan, RK. Watchful waiting in benign prostatic hyperplasia. Curr Opin Urol. 2009;19(1):3-6. doi: 10.1097/ MOU.0b013e32831a6ec6.

9. Oelke M, Giuliano F, Baygani SK, Melby T, Sontag A. Treatment satisfaction with tadalafil or tamsulosinvs placebo in men with lower urinary tract symptoms (LUTS) suggestive of benign prostatic hyperplasia (BPH): results from a randomised, placebo-controlled study. BJU Int. 2014;114(4):568-75. doi: 10.1111/ bju.12733.

10. Traish AM, Mulgaonkar A, Giordano N. The Dark Side of $5 \alpha$-Reductase Inhibitors' Therapy: Sexual Dysfunction, High Gleason Grade Prostate Cancer and Depression. Korean J Urol. 2014;55(6):367-379. doi: 10.4111/ kju.2014.55.6.367.

11.Elshal AM, Elmansy HM, Elkoushy MA, Elhilali MM. Male sexual function outcome after three laser prostate surgical techniques: a single center perspective. Urology. 2012;80(5):1098-104. doi: 10.1016/ j.urology.2012.08.001.

12. Oelke M, Bachmann A, Descazeaud A, Emberton M, Gravas S, Michel MC. EAU Guidelines on the Treatment and Follow-up of Nonneurogenic Male Lower Urinary Tract Symptoms Including Benign Prostatic Obstruction. Eur Urol. 2013;64(1):118-40. doi: 10.1016/ j.eururo.2013.03.004 